\title{
Seeking heavy Higgs bosons through cascade decays
}

\author{
Baradhwaj Coleppa, ${ }^{1, *}$ Benjamin Fuks, ${ }^{2,3,4, \dagger}$ P. Poulose, ${ }^{5, *}$ and Shibananda Sahoo, ${ }^{5, \S}$ \\ ${ }^{1}$ Physics Discipline, Indian Institute of Technology-Gandhinagar, Palaj Campus, Gujarat 382355, India \\ ${ }^{2}$ Sorbonne Universités, UPMC Univ. Paris 06, UMR 7589, LPTHE, F-75005 Paris, France \\ ${ }^{3}$ CNRS, UMR 7589, LPTHE, F-75005 Paris, France \\ ${ }^{4}$ Institut Universitaire de France, 103 boulevard Saint-Michel, 75005 Paris, France \\ ${ }^{5}$ Department of Physics, Indian Institute of Technology-Guwahati, Assam 781039, India
}

(Received 27 December 2017; published 4 April 2018)

\begin{abstract}
We investigate the LHC discovery prospects for a heavy Higgs boson decaying into the standard model Higgs boson and additional weak bosons. We consider a generic model-independent new physics configuration where this decay proceeds via a cascade involving other intermediate scalar bosons and focus on an LHC final-state signature comprised either of four $b$-jets and two charged leptons or of four charged leptons and two $b$-jets. We design two analyses of the corresponding signals, and demonstrate that a $5 \sigma$ discovery at the $14 \mathrm{TeV}$ LHC is possible for various combinations of the parent and daughter Higgs-boson masses. We moreover find that the standard model backgrounds can be sufficiently rejected to guarantee the reconstruction of the parent Higgs boson mass. We apply our analyses to the Type-II two-Higgs-doublet model and identify the regions of the parameter space to which the LHC is sensitive.
\end{abstract}

DOI: 10.1103/PhysRevD.97.075007

\section{INTRODUCTION}

The discovery of a Higgs boson whose properties are consistent with the expectations of the standard model (SM) has undoubtedly been the triumph of the Large Hadron Collider (LHC) thus far [1-4]. While it is clear that the Higgs boson plays a central role in the breaking of the electroweak symmetry, there is still room for a nonminimal Higgs sector with a more involved $\mathrm{TeV}$ scale structure than what could be expected from the SM alone. Moreover, the proof of existence of the Higgs boson has provided an additional tool to narrow down the possibilities for new physics, additional constraints on the new physics parameter spaces being imposed by enforcing the Higgsboson branching ratio and production cross section predictions to agree with the measured values. However, the Higgs boson could also be a perfect laboratory for uncovering new physics in cases where new heavier particles could decay into it, enhancing its indirect production rate.

\footnotetext{
*baradhwaj@iitgn.ac.in

fuks@lpthe.jussieu.fr

poulose@iitg.ernet.in

shibananda@iitg.ernet.in
}

Published by the American Physical Society under the terms of the Creative Commons Attribution 4.0 International license. Further distribution of this work must maintain attribution to the author(s) and the published article's title, journal citation, and DOI. Funded by SCOAP ${ }^{3}$.
One attractive minimal scenario along these lines is the so-called two-Higgs-doublet model (2HDM) where the SM Higgs sector is extended by a second weak doublet of Higgs fields [5,6], although numerous not so minimal options like the minimal supersymmetric standard model $[7,8]$ or the next-to-minimal supersymmetric standard model [9] fall into that category of models as well. The physical spectrum then (minimally) contains, on top of the standard model Higgs boson $h^{0}$, a heavy scalar field $H^{0}$, a pseudoscalar field $A$ as well as a pair of charged Higgs bosons $H^{ \pm}$. A general feature of heavier Higgs bosons consists in the dominance of Higgs-to-Higgs decays in association with a weak boson as soon as they are kinematically open [10-12]. This has consequently motivated the search for the corresponding signals in LHC data by both the ATLAS [13-16] and CMS [17-19] collaborations, as well as a series of theoretical works both in the 2HDM [20-33] and other (less minimal) new physics models [34-39].

In this work, we generalize this concept of Higgs-toHiggs decays when several weak bosons arise from the cascade [40,41], like when in the 2HDM, the heaviest scalar Higgs boson $H^{0}$ decays via a lighter pseudoscalar state $A$ into a SM Higgs boson, $H^{0} \rightarrow A Z \rightarrow h^{0} Z Z$. We further consider SM boson decays into leptons or jets originating from the fragmentation of $b$-quarks, as the latter consists of the dominant decay mode of the SM Higgs boson. In particular, we focus on a final-state signature made of either four leptons and two b-jets, or of two leptons and four $b$-jets, and we analyze the corresponding LHC 
prospects. We first consider a simplified model approach (Sec. II) that can easily be reinterpreted into numerous models featuring an extended Higgs sector. In Sec. III, we take the example of the Type-II 2HDM and translate our findings in the corresponding parameter space. We summarize our work and present our conclusions in Sec. IV.

\section{A SIMPLIFIED MODEL FOR ANALYSING HIGGS CASCADE DECAY SIGNALS}

\section{A. Theoretical framework, benchmark scenarios and simulation setup}

In order to determine the LHC sensitivity to Higgs-toHiggs cascade decays, we make use of a simplified model where the SM is minimally extended in terms of new particles and couplings. In practice, we complement the SM field content by two additional scalar bosons, so that the scalar part of the particle spectrum now contains the observed SM-like Higgs boson $h^{0}$ and two new states that we denote by $H_{1}$ and $H_{2}$. In our convention, $H_{1}$ is the lighter boson and $H_{2}$ the heavier one, and the couplings of the new scalars to the $Z$-boson are kept generic. Whilst their strengths are in principle free parameters, they are traded, in the analyses of the next subsections, for the signal cross sections (see below for more details). We assume varied mass differences between the two new states and the SM Higgs boson, so that we define four different scenarios that we name BP1, BP2, BP3 and BP4 and for which the heavy scalar masses $m_{H_{1}}$ and $m_{H_{2}}$ read

$$
\begin{array}{ll}
\text { BP1: } m_{H_{1}}=250 \mathrm{GeV}, & m_{H_{2}}=400 \mathrm{GeV}, \\
\text { BP2: } m_{H_{1}}=600 \mathrm{GeV}, & m_{H_{2}}=1000 \mathrm{GeV}, \\
\text { BP3: } m_{H_{1}}=250 \mathrm{GeV}, & m_{H_{2}}=1000 \mathrm{GeV}, \\
\text { BP4: } m_{H_{1}}=400 \mathrm{GeV}, & m_{H_{2}}=600 \mathrm{GeV} .
\end{array}
$$

This choice of benchmark points allows us to capture various features that could arise from distinct mass-splitting options. In the BP1 scenario, there is not much available phase space for both the $H_{2} \rightarrow Z H_{1}$ and $H_{1} \rightarrow h^{0} Z$ decays and thus these occur close to threshold. In contrast, the larger mass splittings featured by the $\mathbf{B P} 2$ configuration, in which $m_{H_{2}} \gg m_{H_{1}} \gg m_{h}^{0}$, implies that both the $H_{1}$ and $H_{2}$ decays proceed far from threshold, the decay products being thus expected to feature a larger amount of transverse momentum $p_{T}$. The third scenario BP3 consists of an intermediate case where only the $H_{1} \rightarrow Z h^{0}$ decay occurs close to threshold. Finally, in the fourth scenario BP4, both decays occur far from threshold, but the mass splitting is reduced compared to the $\mathbf{B P 2}$ case.

The different mass splittings between the $h^{0}, H_{1}$ and $H_{2}$ states probed in our benchmarks are expected to impact the kinematic properties of the leptons and $b$-jets originating from the decays of the final-state SM Higgs boson and $Z$-bosons. As a consequence, their study could provide handles for unraveling new physics at the LHC. In the following, we consider the production of the heaviest Higgs boson $\mathrm{H}_{2}$ through gluon fusion, and its subsequent decays into lighter Higgs states and Z-bosons,

$$
p p \rightarrow H_{2} \rightarrow H_{1} Z \rightarrow h^{0} Z Z
$$

Whilst we focus on the dominant Higgs boson decay mode $h^{0} \rightarrow b \bar{b}$, we consider Z-boson decays into a lepton pair $Z \rightarrow \ell^{+} \ell^{-}$and into a bottom-antibottom pair $Z \rightarrow b \bar{b}$. Omitting a final-state signature comprised of six $b$-jets, given the huge associated multijet background and the difficulties induced by the combinatorics to reconstruct all intermediate particles, the final-state signatures of interest therefore consist of a system made either of four leptons and two $b$-jets $(4 \ell 2 b)$ or of two leptons and four $b$-jets $(2 \ell 4 b)$.

As above-mentioned, the signal cross section is taken as a free parameter which correspondingly allows us to ignore the actual strengths of the $Z$-boson couplings to the new scalar bosons. As a benchmark, we make use of a fiducial signal cross sections $\sigma_{\text {fid }}$ fixed to

$\sigma_{\text {fid }}\left(p p \rightarrow H_{2} \rightarrow H_{1} Z \rightarrow h^{0} Z Z \rightarrow 4 \ell 2 b\right)=5 \mathrm{fb}$ and

$\sigma_{\text {fid }}\left(p p \rightarrow H_{2} \rightarrow H_{1} Z \rightarrow h^{0} Z Z \rightarrow 2 \ell 4 b\right)=5 \mathrm{fb}$,

which consist of values lying in the ball park of what could be achieved in a phenomenologically-viable model.

Hard-scattering signal events at a collision center-ofmass energy of $14 \mathrm{TeV}$ are generated by means of the MADGRAPH5_aMC@NLO platform [42]. Practically, we convolute the signal leading-order matrix elements, as automatically obtained from the 2HDM UFO [43] model available from the FEYNRULES repository [44,45], with the leading-order set of NNPDF parton densities version 3.0 [46]. The dependence on the numerical values of the different coupling strengths being factorized out by an appropriate choice of the fiducial cross section, the sole model dependence consists of the Lorentz structure of the various interactions of the $Z$-boson with Higgs bosons. This restriction is however compatible with a large variety of popular multi-Higgs models. The simulation of the SM background proceeds analogously, using instead the Standard Model UFO library shipped with MADGRAPH5_aMC@NLO.

The simulation of the parton showering and hadronization is performed by means of the PYTHIA 6 program [47], and we include the simulation of the response of a typical LHC detector as modeled by DELPHES 3 [48], relying on the CMS-MA5tune parameterization of the detector [49]. The resulting detector-level objects are then reconstructed by applying the anti- $k_{T}$ jet algorithm [50]. More precisely, this is achieved by making use of the MADANALYSIS 5 framework $[51,52]$ to simulate the detector effects and reconstruct the events (through an interface to FASTJET [53]), such 
TABLE I. Leading-order cross section for the different SM processes contributing to the background of our $4 \ell+2 b$ analysis. They include the relevant branching rations and the preselection cuts of Eqs. (2.5) and (2.6).

\begin{tabular}{lccccc}
\hline \hline Background & $Z Z b \bar{b}$ & $t \bar{t} Z$ & $t \bar{t} W$ & $W W Z b \bar{b}$ & $W W W b \bar{b}$ \\
\hline$\sigma \times \mathrm{BR}$ & $0.1 \mathrm{fb}$ & $1.2 \mathrm{fb}$ & $2.3 \mathrm{fb}$ & $1.2 \mathrm{fb}$ & $2.1 \mathrm{fb}$ \\
\hline \hline
\end{tabular}

a framework being also used to implement the analyses described in the next subsections.

\section{B. Probing Higgs cascades in the $4 \mathscr{\ell} 2 \boldsymbol{b}$ final state}

In this section, we focus on the process of Eq. (2.2) when both Z-bosons decay leptonically,

$$
p p \rightarrow H_{2} \rightarrow H_{1} Z \rightarrow h^{0} Z Z \rightarrow b \bar{b} \ell_{1}^{+} \ell_{1}^{-} \ell_{2}^{+} \ell_{2}^{-} .
$$

The signal under consideration is thus made of one pair of $b$-jets and two pairs of opposite-sign same-flavor leptons. In our analysis, we restrict ourselves to lepton and jet candidates whose transverse momentum $\left(p_{T}^{\ell}\right.$ and $\left.p_{T}^{j}\right)$ and pseudorapidity $\left(\eta^{\ell}\right.$ and $\left.\eta^{j}\right)$ satisfy

$$
\begin{aligned}
p_{T}^{j} & >20 \mathrm{GeV}, \quad p_{T}^{\ell}>10 \mathrm{GeV}, \\
\left|\eta^{j}\right| & <5 \quad \text { and } \quad\left|\eta^{\ell}\right|<2.5 .
\end{aligned}
$$

Moreover, we omit from the analysis any pair of jet candidates that would not be well separated from each other as well as any lepton that would be too close to a jet. In practice, we impose that the angular distance in the transverse plane between two jets $\left(\Delta R_{j j}\right)$ and the one between a jet and a lepton $\left(\Delta R_{\ell j}\right)$ satisfy

$$
\Delta R_{j j}>0.4 \text { and } \Delta R_{\ell j}>0.4 .
$$

The dominant contributions to the SM background hence arise from $Z Z b \bar{b}, t \bar{t} V$ and $W W V b \bar{b}$ production, with $V$ being a $W$-boson or a $Z$-boson. Including the branching

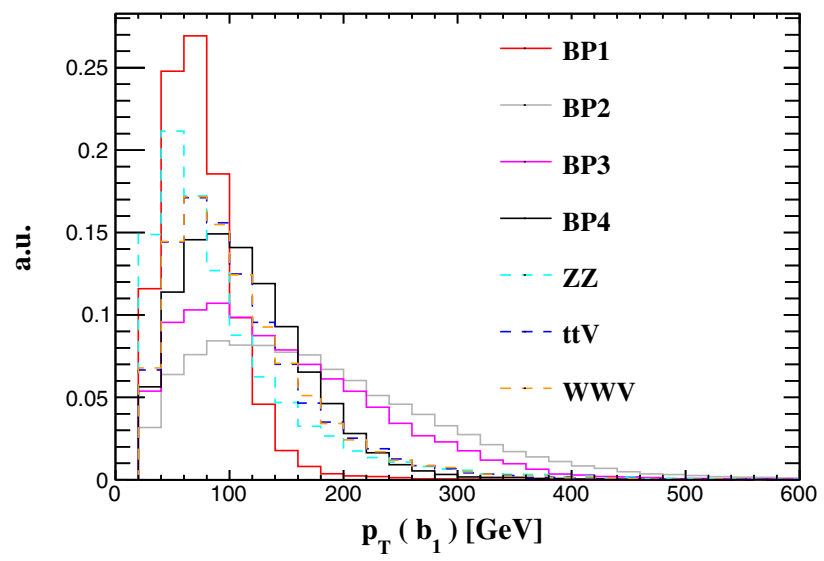

ratio (BR) corresponding to the $4 \ell 2 b$ final state, the leading-order cross sections as returned by MADGRAPH5_aMC@NLO are given in Table I.

We implement a flavor-blind analysis in order to increase the signal statistics, although we ignore tau leptons as those objects are more complicated to reconstruct. We hence focus on leptons of the first two generations, so that $\ell_{1}$, $\ell_{2}=e, \mu$ in Eq. (2.4), and we require the presence of two positively-charged and two negatively-charged leptons,

$$
N\left(\ell^{+}\right)=N\left(\ell^{-}\right)=2 .
$$

The corresponding signal selection efficiency is about $40 \%$, many leptons being missed as lying outside the acceptance of the detector or being too soft for being correctly reconstructed. The corresponding background rejection factor is slightly below 7, as many of the background components do not yield a tetraleptonic signal.

Although the signal is expected to feature the presence of two $b$-jets, $b$-tagging is not perfect. Harder $b$-jets are indeed more easily correctly reconstructed than softer $b$-jets. The transverse momentum distributions of the two leading $b$-tagged jets is illustrated in Fig. 1, in which we can observe that the bulk of the events feature softer $b$-jets. Moreover, for scenarios where the mass splitting between the Higgs bosons is large, the produced SM Higgs boson is often boosted. The two $b$-jets are therefore not resolved, and a single $b$-jet is instead reconstructed. It consequently turns out that only $10-20 \%$ of the surviving signal events contain two tagged $b$-jets. For the BP2 and BP4 scenarios, the mass splittings between the different Higgs states is large and $b$-jets are more efficiently tagged, the signal selection efficiency being larger. In contrast, the selection efficiency is found to be smaller for the two other scenarios, as the $H_{1} \rightarrow h^{0} \mathrm{Z}$ decay proceeds almost at threshold.

On the other hand, $40 \%-50 \%$ of the signal events are tagged as single- $b$-jet events, and a significant fraction of them do not feature any tagged $b$-jets at all. In order to

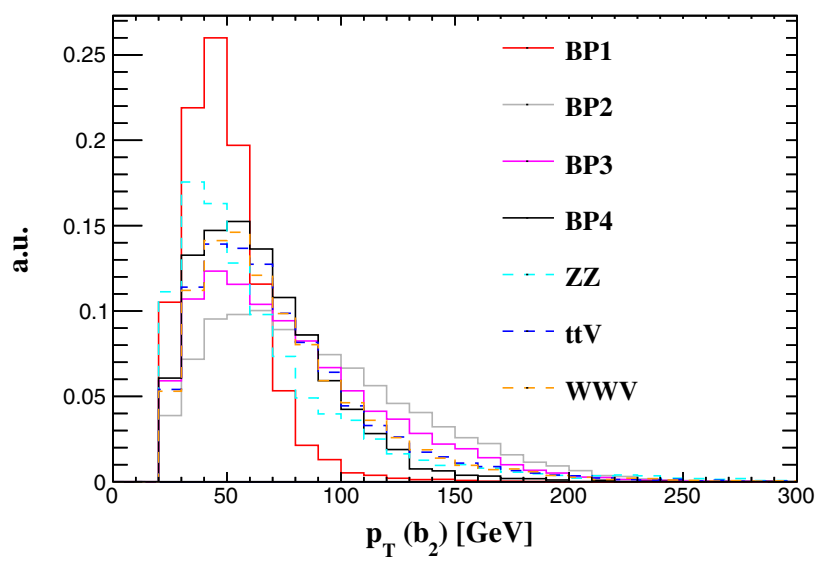

FIG. 1. Normalized transverse-momentum distribution of the leading (left) and next-to-leading (right) $b$-tagged jets after having selected events featuring two pairs of charged leptons. 
TABLE II. Number of events surviving each selection step for the four considered benchmark scenarios, as well as for the SM background. The results are normalized to an integrated luminosity of $1000 \mathrm{fb}^{-1}$ and include a conservative $K$-factor of 2 for the background. Results are presented for the $N(b)=2$ signal region (upper table) and $N(b)=1$ signal region (lower table).

\begin{tabular}{lcrrrrr}
\hline \hline & Selection step & BP1 & BP2 & BP3 & BP4 & Background \\
\hline 0 & Initial & 5000 & 5000 & 5000 & 5000 & 13636 \\
1 & $N\left(\ell^{+}\right)=N\left(\ell^{-}\right)=2$ & 1993 & 2723 & 1979 & 2373 & 1992 \\
2 & $N(b)=2$ & 206 & 490 & 260 & 340 & 231 \\
3 & $E_{T}<50 \mathrm{GeV}$ & 203 & 415 & 220 & 321 & 66 \\
4 & $90 \mathrm{GeV}<M_{b b}<150 \mathrm{GeV}$ & 160 & 344 & 174 & 257 & 16 \\
5 & $p_{T}\left(\ell_{1}\right)>75 \mathrm{GeV}$ & $\sim 0$ & 200 & 59 & 37 & 2 \\
& $p_{T}\left(\ell_{2}\right)>50 \mathrm{GeV}$ & & & & & \\
\hline
\end{tabular}

\begin{tabular}{lcrrrrr}
\hline \hline & Selection step & BP1 & BP2 & BP3 & BP4 & Background \\
\hline 0 & Initial & 5000 & 5000 & 5000 & 5000 & 13636 \\
1 & $N\left(\ell^{+}\right)=N\left(\ell^{-}\right)=2$ & 1993 & 2723 & 1979 & 2373 & 1992 \\
2 & $N(b)=1$ & 884 & 1310 & 910 & 1115 & 818 \\
3 & $E_{T}<50 \mathrm{GeV}$ & 871 & 1122 & 782 & 1060 & 242 \\
4 & $p_{T}\left(\ell_{1}\right)>75 \mathrm{GeV}$ & $\sim 0$ & 650 & 296 & 163 & 20 \\
& $p_{T}\left(\ell_{2}\right)>50 \mathrm{GeV}$ & & & & & \\
\hline \hline
\end{tabular}

recover the large number of signal events featuring a single $b$-jet, we consider two independent signal regions in which we respectively require 2 and $1 b$-tagged jet,

$$
N(b)=2 \text { or } \quad N(b)=1 .
$$

This cut allows for reducing the background by a factor of about 10 and 2.5 in the two and one jet cases respectively.

As shown in Table II for an integrated luminosity of $1000 \mathrm{fb}^{-1}$, about 200-500 and 900-1300 signal events are expected to respectively populate the $N(b)=2$ and $N(b)=1$ signal regions, to be compared with 230 and 800 background events (including a conservative $K$-factor

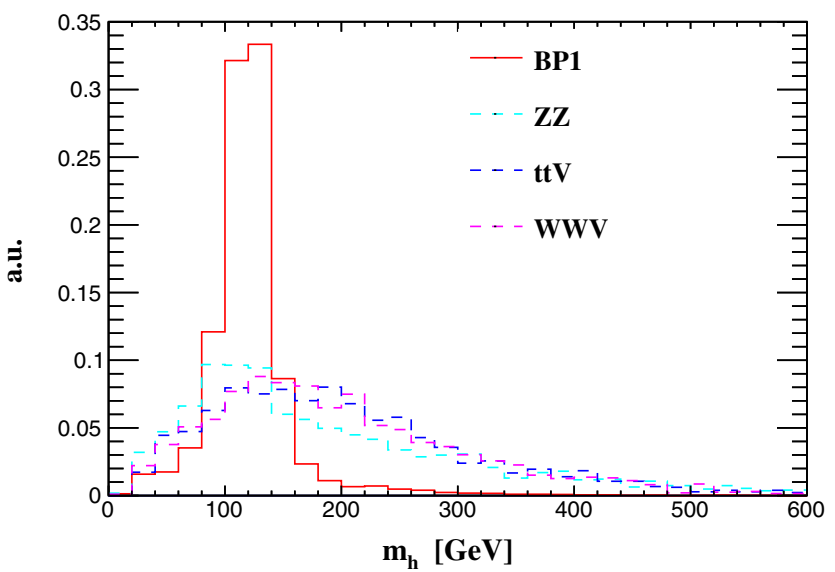

of 2). From this stage, background rejection can be improved by restricting the missing transverse energy $\mathbb{E}_{T}$ in the event,

$$
\mathbb{E}_{T}<50 \mathrm{GeV}
$$

This selection leaves the signal barely unaffected as it is expected to be fully visible, and reduces the background by an extra factor of 3 . The surviving background events are mostly originating from $t \bar{t} Z$ and $W W Z b \bar{b}$ production. In the $N(b)=2$ signal region, an extra selection can be imposed as the invariant mass of the dijet system $M_{b b}$ has to be compatible with the mass of the Higgs boson,

$$
90 \mathrm{GeV}<M_{b b}<150 \mathrm{GeV} .
$$

The importance of this last selection is demonstrated in Fig. 2 for the BP1 and BP2 scenarios, where the distributions in the invariant mass of the system made of the two leading $b$-tagged jets is shown for two representative benchmark scenarios, and the main contribution to the background. The availability of reconstructing the standard model Higgs boson is hence crucial when searching for heavier Higgs bosons, and achievable even for compressed spectra.

Finally, we make use of the different properties of the leading lepton $\ell_{1}$ and next-to-leading lepton $\ell_{2}$ for the signal and the background (as illustrated in Fig. 3 for the BP2 scenario) to further improve the sensitivity, enforcing

$$
p_{T}\left(\ell_{1}\right)>75 \mathrm{GeV} \text { and } p_{T}\left(\ell_{2}\right)>50 \mathrm{GeV} \text {. }
$$

These two last cuts yield a basically background-free environment. The corresponding signal selection efficiencies are usually large, except for scenarios featuring a small mass splitting such as in the BP1 configuration. We will

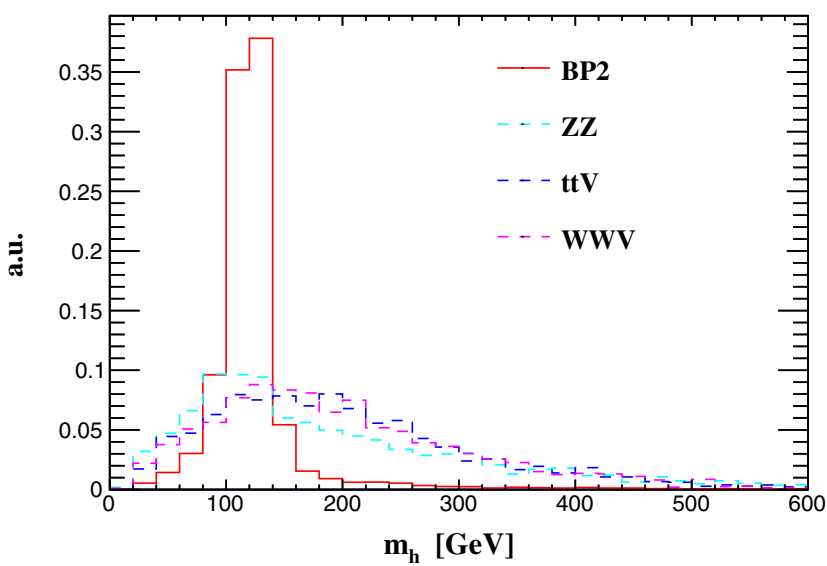

FIG. 2. Normalized invariant-mass spectrum of the system comprised of the two leading $b$-jets, in the context of the $N(b)=2$ analysis. Results are shown for both the signal corresponding to the BP1 (left) and BP2 (right) scenarios, and for the dominant contributions to the background. 

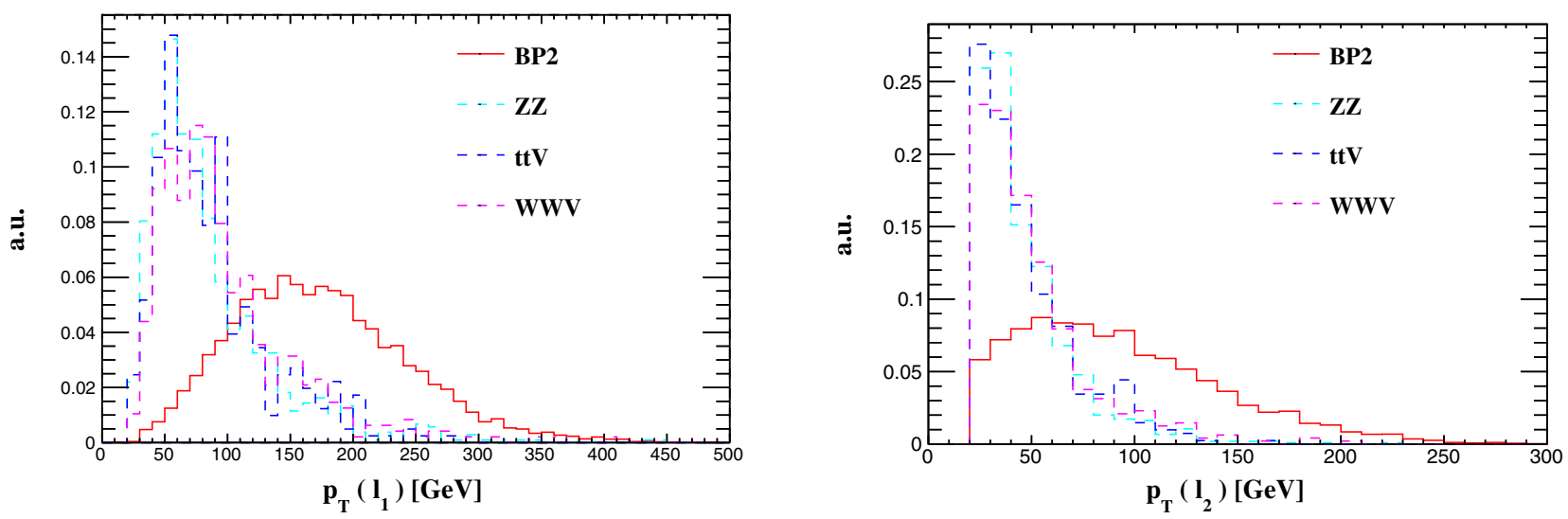

FIG. 3. Normalized $p_{T}$ distributions of the leading (left) and next-to-leading (right) leptons, both for the signal corresponding to the BP2 scenario and for the dominant background contributions, in the case of the $N_{b}=2$ signal region.

therefore ignore this cut for what concerns the BP1 configuration.

In addition to the conservative $K$-factor of 2 that has been included in the background numbers to model higher-order effects, we assess the potential effects of the systematic uncertainties of $x \%$ by computing the signal significance as [54]

$$
\begin{aligned}
Z= & \sqrt{2}\left((S+B) \ln \left[\frac{(S+B)\left(B+\Delta_{B}^{2}\right)}{B^{2}+(S+B) \Delta_{B}^{2}}\right]\right. \\
& \left.-\frac{B^{2}}{\Delta_{B}^{2}} \ln \left[1+\frac{\Delta_{B}^{2} S}{B\left(B+\Delta_{B}^{2}\right)}\right]\right)^{\frac{1}{2}} \quad \text { with } \quad \Delta_{B}=x B .
\end{aligned}
$$

In Fig. 4, we present the LHC sensitivity, as defined by Eq. (2.12), to the signal for the different considered benchmark scenarios and for both the $N(b)=2$ and $N(b)=1$ signal regions. The normalization moreover corresponds to an integrated luminosity of $1000 \mathrm{fb}^{-1}$.

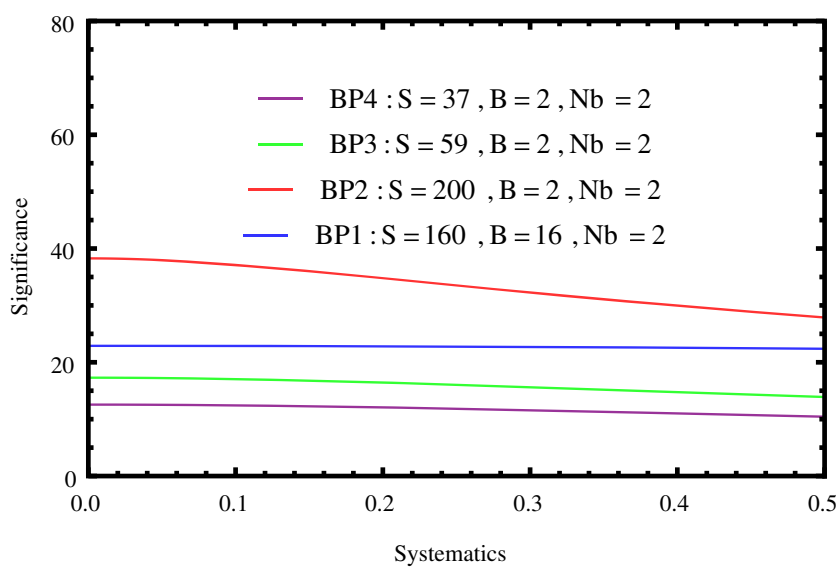

All the selection cuts introduced above have been applied, with the exception of the one on the leading and next-toledaing leptons in the case of the BP1 scenario, as indicated above. The results are shown for various levels of systematic uncertainties ranging from $0 \%$ to $50 \%$. They are found stable with respect to the systematics thanks to a very large signal and the almost background-free environment originating from our selection.

The results presented so far correspond to a signal cross section that has been fixed to $5 \mathrm{fb}$. In Fig. 5, we relax this hypothesis and show the dependence of the significance on the number of signal events $S$ when $10 \%$ of systematic uncertainties is assumed. We again consider both the $N(b)=2$ (left) and $N(b)=1$ (right) signal regions, and study the dependence on the last cut on the transverse momentum of the two leading leptons. As expected, the effect of this selection increases the significance for a given number of signal events. Conversely, while about 25 and 85 signal events are required for a $5 \sigma$ discovery without imposing any requirement on the leptons, for the $N(b)=2$

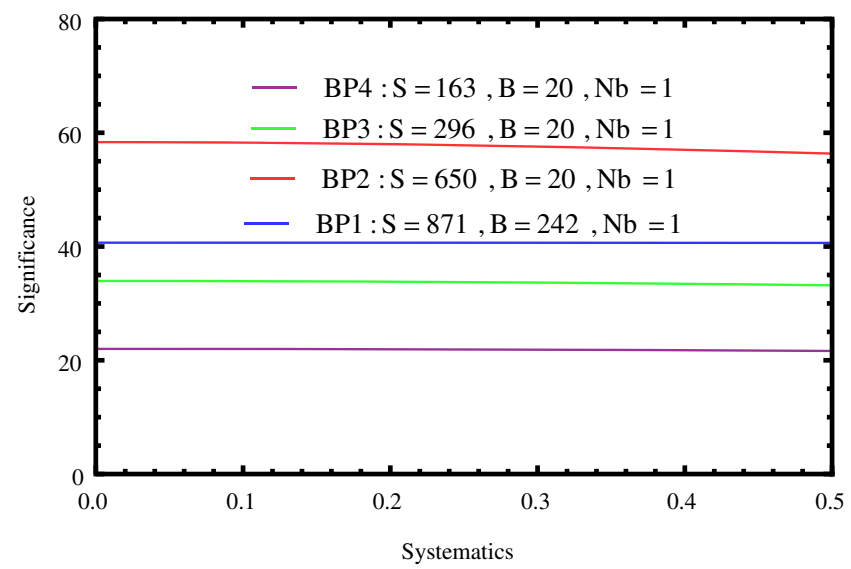

FIG. 4. LHC significance, as defined by Eq. (2.12), to the considered Higgs cascade decays for the four considered benchmark scenarios and assuming a luminosity of $1000 \mathrm{fb}^{-1}$. We show results for the $N(b)=2$ (left) and $N(b)=1$ (right) signal regions, and calculate the dependence of the significance on the level of systematic uncertainties taken as $\Delta_{B}=x B$ (with $x$ being shown on the $x$-axis). 

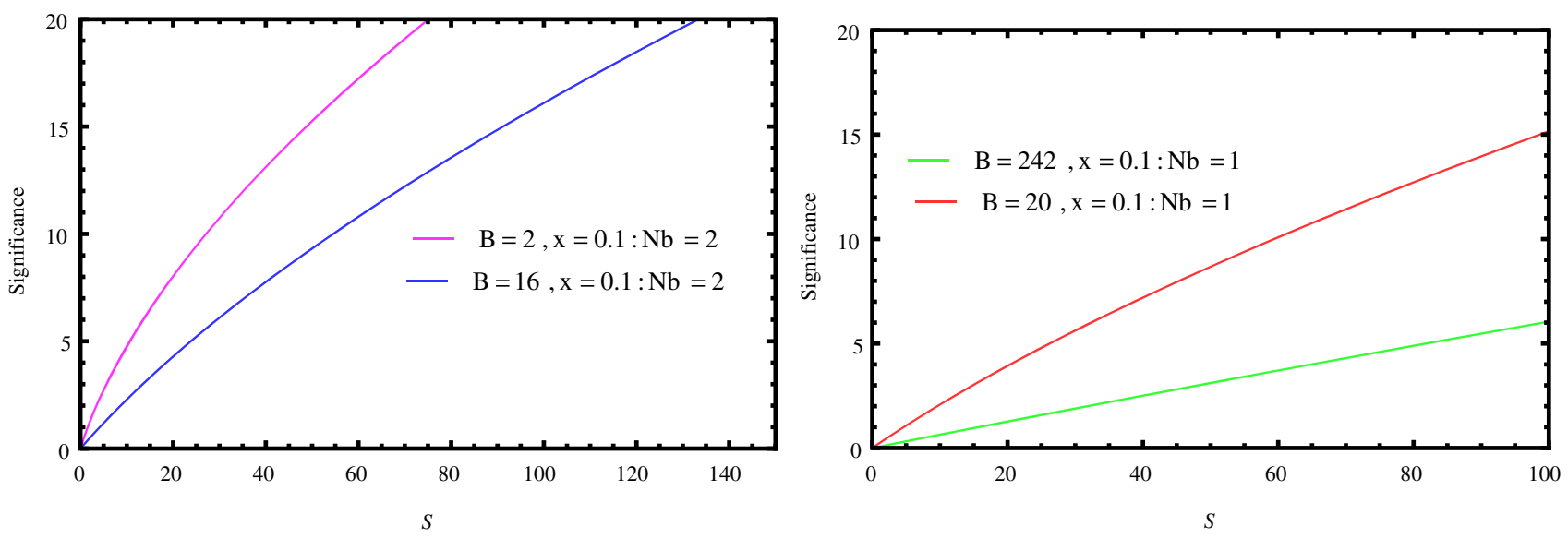

FIG. 5. Variation of the significance with respect to the number of signal events $S$ for the $N(b)=2$ (left) and $N(b)=1$ (right) signal regions, both when the cut on the transverse momentum of the leading and next-to-leading leptons is applied (purple and red) and ignored (blue and green). We consider a level of systematic uncertainties of $10 \%$.

and $N(b)=1$ signal regions respectively, these numbers are reduced to 10 and 25 after constraining the transverse momentum of the leptons as in Eq. (2.11).

Translating these numbers in term of cross section, compressed scenarios like our BP1 configuration could yield an observable signal in the $N(b)=2$ and $N(b)=1$ signal regions as long as the production rate is at least $0.78 \mathrm{fb}$ and $0.49 \mathrm{fb}$, respectively. The reach of the $N(b)=1$ signal region is found to be larger, by virtue of the efficiency to correctly identify one $b$-jet that is larger than the one to identify two $b$-jets. The two regions are however complementary, as even if the $N(b)=1$ region is better for what concerns the reach, the $N(b)=2$ analysis offers a way to uniquely reconstruct the intermediate heavy Higgs states as illustrated in Fig. 6. For scenarios exhibiting a mass spectrum featuring larger splittings like in the BP2 configuration, the final-state objects are harder, which implies a better reconstruction efficiency. Accordingly,

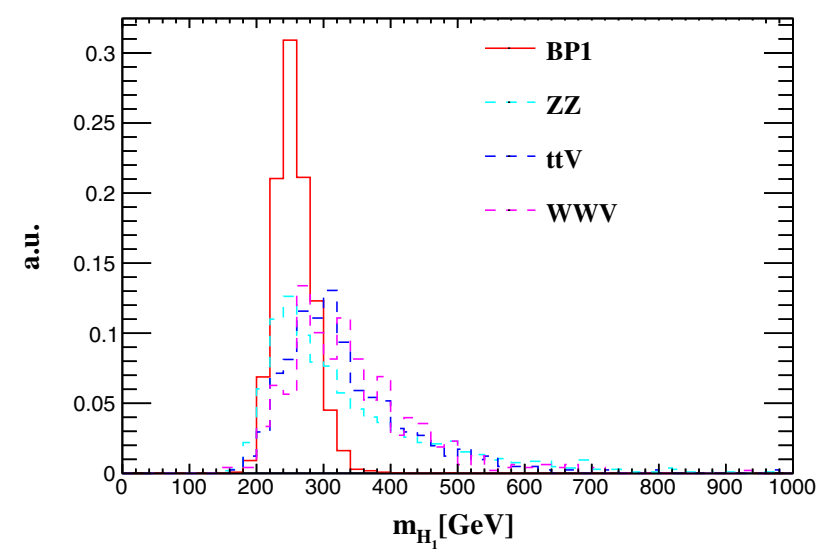

one obtains better expected limits on the production rate, the observable cross section being $0.25 \mathrm{fb}$ and $0.19 \mathrm{fb}$ in the $N(b)=2$ and $N(b)=1$ analysis, respectively. For scenarios featuring a smaller mass splitting like in the BP3 case (where one of the decays has to occur close to threshold) or in the BP4 case (where both decays have less available phase space than in the BP2 case), the final-state objects are softer, which results in degraded expected limits on the signal cross section, but still in the $1 \mathrm{fb}$ regime.

\section{Probing Higgs cascades in the $2 \ell \boldsymbol{\ell} b$ final state}

The Higgs cascade signal that we consider in this work could also give rise to a final-state signature comprised of four $b$-jets and one pair of opposite-sign leptons of the same flavor,

$$
p p \rightarrow H_{2} \rightarrow H_{1} Z \rightarrow h^{0} Z Z \rightarrow b \bar{b} b \bar{b} \ell^{+} \ell^{-} .
$$

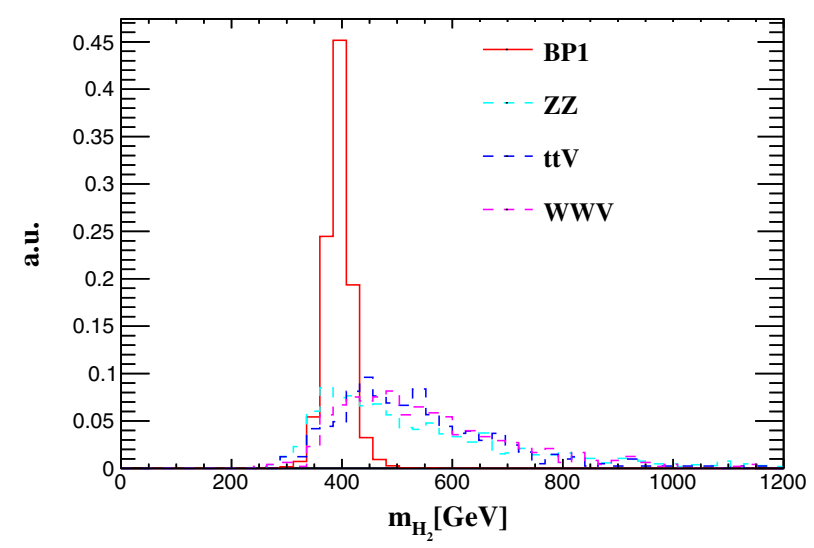

FIG. 6. Normalized invariant-mass spectrum for the intermediate $H_{1}$ (left) and $H_{2}$ states in the context of the $N(b)=2$ analysis (the last cut being omitted) and for the BP1 scenario. The results are derived from the reconstruction of the $2 b \ell^{+} \ell^{-}$and $2 b 4 \ell$ systems. Whilst the spread in the $H_{1}$ invariant mass spectrum stems from the different possible combinations of the leptons, the distribution is found similar to the one obtained for any other lepton combination. 
TABLE III. Leading-order cross section for the different SM processes contributing to the background of our $2 \ell+4 b$ analysis. They include the relevant branching ratios and the preselection cuts of Eqs. (2.5) and (2.6).

\begin{tabular}{lccc}
\hline \hline Background & $\ell^{+} \ell^{-}+$jets & $W^{+} W^{-}+$jets & $t \bar{t} h^{0}$ \\
\hline$\sigma \times \mathrm{BR}$ & $3.2 \mathrm{pb}$ & $109.1 \mathrm{fb}$ & $14 \mathrm{fb}$ \\
\hline \hline
\end{tabular}

The combinatorics induced by the final-state reconstruction and the more abundant SM background renders the task of discriminating the signal from the background complicated. We however verify, in this section, the existence of any potential corresponding handle. The dominant contributions to the SM background arise from the associated production of a Drell-Yan pair of leptons with jets, $W$-boson pair production with jets and $t \bar{t} h^{0}$ production. The leading-order cross sections as returned by MADGRAPH5_aMC@NLO are shown in Table III.

In our analysis, jet and lepton candidates are selected as in Eqs. (2.5) and (2.6). We preselect events containing one positively-charged and one negatively-charged lepton regardless of the lepton flavor,

$$
N\left(\ell^{+}\right)=N\left(\ell^{-}\right)=1,
$$

and we require in addition the presence of at least four jets out of which three should be $b$-tagged,

$$
N(j) \geq 4 \quad \text { with } \quad N(b)=3 .
$$

Whilst four $b$-tagged jets are expected, the loss in signal efficiency induced by the requirement of a fourth $b$-tag would make the signal unobservable (see the discussion in Sec. II B). The combined signal efficiency for these preselection cuts is of about 5\%-6\%, for a background rejection factor of about 250 . We then impose the lepton pair to be compatible with the decay of a Z-boson, constraining its invariant mass $M_{\ell \ell}$ to satisfy

$$
80 \mathrm{GeV} \leq M_{\ell \ell} \leq 100 \mathrm{GeV} .
$$

This allows for the reduction of the diboson and Higgs backgrounds without impacting the signal too much. At this stage, the number of background events is of about 13000, while the number of signal events is expected to be in the 200-450 window for the different scenarios, as illustrated in Table IV. The signal is thus not visible over the background.

The fact that all final-state objects are not fully identified and the combinatorics that may result from the reconstruction of the intermediate $H_{1}$ and $Z$-bosons make a kinematic fit complicated, in particular once detector effects are accounted for. We therefore approximate the invariant-mass spectrum of the $\mathrm{H}_{2}$ boson by the invariantmass distribution of the system comprised of the four leading jet candidates and the selected pair of leptons, $M_{\mathrm{H}_{2}}$. As illustrated in Fig. 7, this variable serves as a good discriminator of the signal from the background. The distribution turns to be very broad for some scenarios, the distortion being larger for new physics scenarios featuring larger mass splittings as this configuration could induce extra radiation and thus more jets in the final state. There is no perfect scenario-independent selection that would allow for the observation of the signal from the overwhelming background. Such a potential cut indeed strongly depends on the mass splittings between the different Higgs states. We therefore propose four different cuts,

(5a) $300 \mathrm{GeV}<M_{H_{2}}<500 \mathrm{GeV}$,

(5b) $900 \mathrm{GeV}<M_{H_{2}}<1400 \mathrm{GeV}, \quad p_{T}^{\ell}>70 \mathrm{GeV}$,

(5c) $900 \mathrm{GeV}<M_{H_{2}}<1400 \mathrm{GeV}, \quad p_{T}^{\ell}>60 \mathrm{GeV}$,

(5d) $500 \mathrm{GeV}<M_{H_{2}}<700 \mathrm{GeV}, \quad p_{T}^{\ell}>50 \mathrm{GeV}$,

where the extra selection on the leptons allow for a better signal discrimination in the case of a not too light spectrum

TABLE IV. Number of events surviving each selection step for the four considered benchmark scenarios, as well as for the SM

\begin{tabular}{|c|c|c|c|c|c|c|}
\hline & Selection step & BP1 & BP2 & BP3 & BP4 & Background \\
\hline$\overline{0}$ & Initial & 5000 & 5000 & 5000 & 5000 & $6.657 \times 10^{6}$ \\
\hline 1 & $N\left(\ell^{+}\right)=N\left(\ell^{-}\right)=1$ & 2815 & 3006 & 2747 & 2971 & $3.695 \times 10^{6}$ \\
\hline 2 & $N(j) \geq 4$ & 2811 & 3004 & 2735 & 2970 & $3.644 \times 10^{6}$ \\
\hline 3 & $N(b)=3$ & 228 & 506 & 302 & 394 & 25062 \\
\hline 4 & $80 \mathrm{GeV}<M_{\ell \ell}<100 \mathrm{GeV}$ & 201 & 434 & 258 & 343 & 13072 \\
\hline $5 \mathrm{a}$ & $300 \mathrm{GeV}<M_{H_{2}}<500 \mathrm{GeV}$ & 121 & $\ldots$ & $\ldots$ & $\ldots$ & 1954 \\
\hline $5 b$ & $900 \mathrm{GeV}<M_{H_{2}}<1400 \mathrm{GeV}, p_{T}^{\ell}>70 \mathrm{GeV}$ & $\cdots$ & 192 & .. & $\cdots$ & 455 \\
\hline $5 \mathrm{c}$ & $900 \mathrm{GeV}<M_{H_{2}}<1400 \mathrm{GeV}, p_{T}^{\ell}>60 \mathrm{GeV}$ & $\cdots$ & $\cdots$ & 94 & $\cdots$ & 649 \\
\hline $5 d$ & $500 \mathrm{GeV}<M_{H_{2}}<700 \mathrm{GeV}, p_{T}^{\ell}>50 \mathrm{GeV}$ & $\cdots$ & $\cdots$ & $\cdots$ & 91 & 552 \\
\hline
\end{tabular}
background. The results are normalized to an integrated luminosity of $1000 \mathrm{fb}^{-1}$ and include a conservative $K$-factor of 2 for the background. 


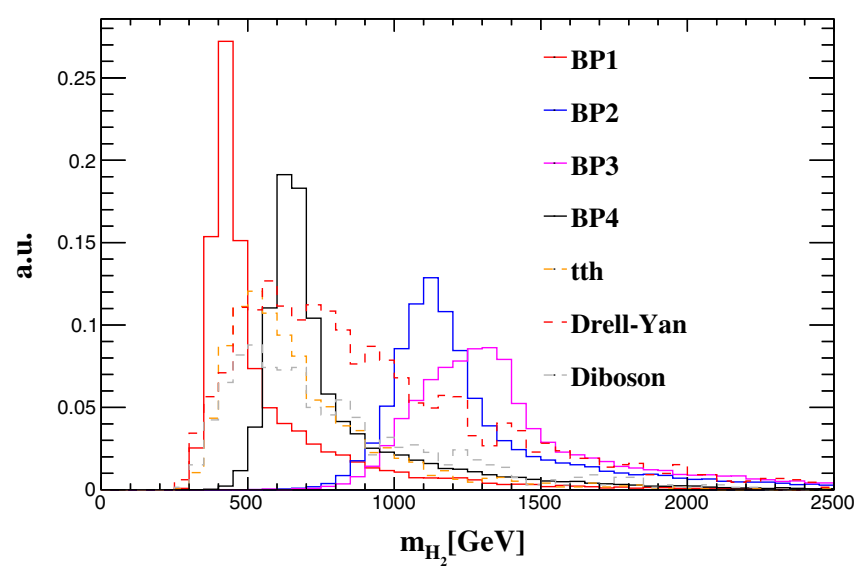

FIG. 7. Normalized invariant-mass distribution of the heavy $\mathrm{H}_{2}$ boson for the main contributions to the SM background and the four signal scenarios, once all other selection cuts have been applied.

(as this yields harder leptons). The first of these selection target setups similar to the configuration of the BP1 scenario where the spectrum is compressed and light, while the second selection aims for scenarios featuring heavier Higgs boson with enough mass splittings to guarantee the presence of very hard leptons in the final state. The third choice is also appropriate for heavier spectra, but it potentially allows for intermediate decays being close to threshold. Finally, the last selection targets spectra where the Higgs bosons are not too heavy but where the decays can occur far from threshold.

For an integrated luminosity of $1000 \mathrm{fb}^{-1}$, these cuts lead to an LHC sensitivity to the BP1, BP2, BP3 and BP4 scenarios of $2.7 \sigma, 8.5 \sigma, 3.6 \sigma$ and $3.8 \sigma$ respectively, when $10 \%$ of systematic uncertainties is also factored in. These results are however found not to depend on the systematics. Although potentially promising, the $4 b 2 \ell$ signature does not provide as clear a handle on the signal as the $2 b 4 \ell$ channel and will therefore be not considered in what follows.

\section{MODEL IMPLICATIONS}

We now turn to the understanding of the implications of the analyses that have been designed in Sec. II in a simplified model context. We investigate below how the simplified spectra introduced in the previous section can be realized in a concrete model with an enlarged scalar spectrum, and investigate the reach of our analysis. As an operating example, we choose the Type-II 2HDM. For details about the model and the couplings, we refer to Ref. [6] and to Sec. III A where we sketch the essential details. Our phenomenological results are given in Sec. III B.

\section{A. The two-Higgs-doublet model-Spectrum and couplings}

The 2HDM has been extensively studied during the last decades, both as a standalone model and also often as the scalar sector of a larger model like the minimal supersymmetric standard model (MSSM). Unlike the SM, the $2 \mathrm{HDM}$ contains two weak doublets of Higgs fields $\phi_{1}$ and $\phi_{2}$ of opposite hypercharge $Y= \pm 1 / 2$. At the minimum of the potential, the neutral components of both doublets develop vacuum expectation values (vev),

$$
\left\langle\phi_{1}^{0}\right\rangle=\frac{1}{\sqrt{2}} v_{1} \quad \text { and } \quad\left\langle\phi_{2}^{0}\right\rangle=\frac{1}{\sqrt{2}} v_{2},
$$

where the vev of the SM Higgs fields $v$ is obtained through $v_{1}^{2}+v_{2}^{2} \equiv v^{2}=\left(\sqrt{2} G_{F}\right)^{-1}$ with $G_{F}$ being the Fermi constant. The two vevs $v_{1}$ and $v_{2}$ are thus not arbitrary as their quadratic sum is connected to the mass scale of the electroweak bosons. We have thus here a single free parameter that is often chosen as the ratio $v_{2} / v_{1}=\tan \beta$.

The breaking of the electroweak symmetry induces a mixing of the scalar degrees of freedom that reads, at tree-level,

$$
\begin{aligned}
\left(\begin{array}{c}
H^{0} \\
h^{0}
\end{array}\right) & =\left(\begin{array}{cc}
\cos \alpha & \sin \alpha \\
-\sin \alpha & \cos \alpha
\end{array}\right)\left(\begin{array}{c}
\Re\left\{\phi_{1}^{0}\right\} \\
\Re\left\{\phi_{2}^{0}\right\}
\end{array}\right), \\
A & =-\sin \beta \Im\left\{\phi_{1}^{0}\right\}+\cos \beta \Im\left\{\phi_{2}^{0}\right\}, \\
H^{ \pm} & =-\sin \beta \phi_{1}^{ \pm}+\cos \beta \phi_{2}^{ \pm},
\end{aligned}
$$

where $h^{0}$ and $H^{0}$ are $C P$-even mass-eigenstates, $A$ is a $C P$-odd mass-eigenstate and $H^{ \pm}$are the physical charged Higgs bosons. In the notation of Sec. II, the $H_{2}$ boson can in principle equally be mapped to the heavier scalar state $H^{0}$ or the pseudoscalar state $A$, whereas we impose the lightest $C P$-even state $h^{0}$ to be the SM Higgs boson of mass $m_{h^{0}}=125 \mathrm{GeV}$. While the model features in general many free parameters, they can all be reduced, for our purposes, to the value of the mixing angle $\alpha$ and $\tan \beta$.

The way in which the mixing angles enter the couplings of the Higgs bosons to the SM particles depends on the $2 \mathrm{HDM}$ configuration under consideration. For the sake of the example, we consider in this section the $C P$-conserving version of the Type II $2 \mathrm{HDM}$, where the first Higgs field $\phi_{1}$ couples to the down-type quarks and the charged leptons, and the second Higgs field $\phi_{2}$ couples to the up-type quarks, as in the MSSM.

Whereas two different Higgs cascades can in principle be considered,

$p p \rightarrow H^{0} \rightarrow A Z \rightarrow h^{0} Z Z$ and $p p \rightarrow A \rightarrow H^{0} Z \rightarrow h^{0} Z Z$,

the absence of a $H^{0} h^{0} Z$ coupling in the Type II 2HDM implies that the second of the above processes is forbidden. In the notation of Sec. II, this thus means that $H_{1} \equiv A$ and $H_{2} \equiv H^{0}$. The corresponding production cross section depends on the $\alpha$ and $\beta$ angle through the off-diagonal coupling strengths of the Higgs bosons to the Z-boson $g_{H^{0} A Z}$ and $g_{A h^{0} Z}$, 
$g_{H^{0} A Z}=-\frac{g \sin (\beta-\alpha)}{2 \cos \theta_{w}} \quad$ and $\quad g_{A h^{0} Z}=\frac{g \cos (\beta-\alpha)}{2 \cos \theta_{w}}$,

with $g$ being the weak coupling and $\theta_{w}$ the electroweak mixing angle and the coupling of the Higgs bosons to $t \bar{t}$ and $b \bar{b}$ - see Sec. III B. While other Higgs production process could be relevant as potentially yielding an observable signal (like the vector-boson fusion production of an $A$ boson), we opt to ignore them all as they would require dedicated analyses which goes beyond the scope of this work.

\section{B. Higgs-boson production cross sections and branching ratios}

In order to evaluate the constraints that could be imposed on the 2HDM parameter space from $H^{0}$ cascades, we first need to calculate the $p p \rightarrow H^{0}$ cross section. We make use of the SM results $[55,56]$ that we rescale by an appropriate loop factor,

$\sigma\left(p p \rightarrow H^{0}\right)=\sigma_{\mathrm{SM}} \times \frac{\left|\frac{\sin \alpha}{\sin \beta} F_{1 / 2}^{h}\left(\tau_{t}\right)+\frac{\cos \alpha}{\cos \beta} F_{1 / 2}^{h}\left(\tau_{b}\right)\right|^{2}}{\left|F_{1 / 2}^{h}\left(\tau_{t}\right)+F_{1 / 2}^{h}\left(\tau_{b}\right)\right|^{2}}$,

where $\tau_{f}=4 m_{f}^{2} / m_{H^{0}}^{2}$ (with $\left.f=t, b\right)$ and where the loop function $F_{1 / 2}^{h}$ is given by

$$
\begin{aligned}
& F_{1 / 2}^{h}=-2 \tau[1+(1-\tau) f(\tau)] \\
& f(\tau)= \begin{cases}{\left[\sin ^{-1}(1 / \sqrt{\tau})\right]^{2}} & \tau \geq 1, \\
-\frac{1}{4}\left[\ln \frac{1+\sqrt{1-\tau}}{1-\sqrt{1-\tau}}-i \pi\right]^{2} & \tau<1 .\end{cases}
\end{aligned}
$$

In Fig. 8 (upper-left panel), we present, in a convenient $(\sin (\beta-\alpha), \tan \beta)$ plane, the dependence on the $H^{0}$ gluon
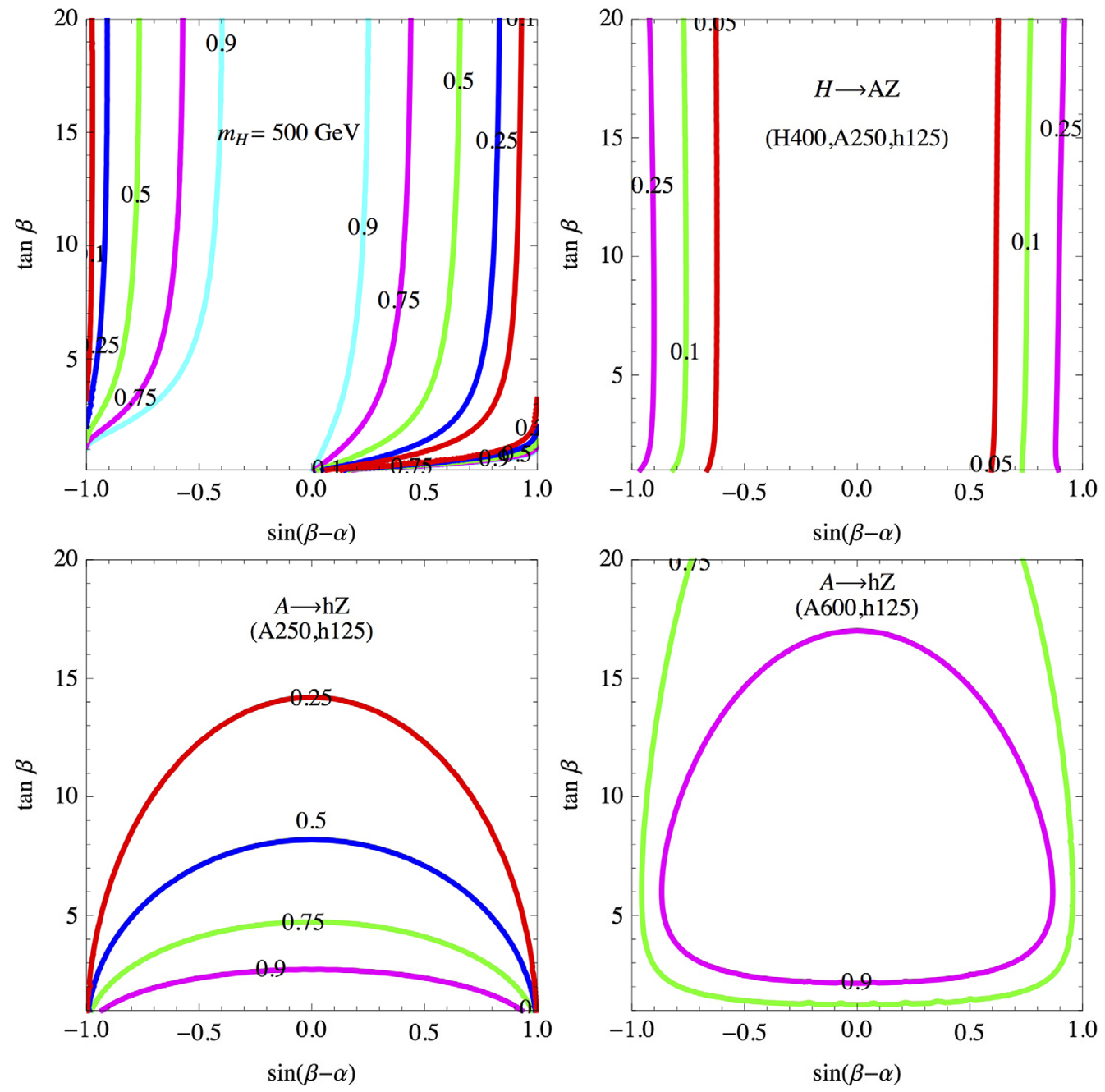

FIG. 8. Dependence of the $\sigma\left(p p \rightarrow H^{0}\right)$ cross section (upper left panel) and the $H^{0} \rightarrow A Z$ (upper right panel) and $A \rightarrow h^{0} Z$ (lower panel) branching ratios on the Higgs mixing angles $\alpha$ and $\beta$. The results are shown in the $(\sin (\beta-\alpha), \tan \beta)$ plane and for the Higgs boson masses introduced in Sec. II. The cross section values (in the upper left figure) are normalized to the corresponding SM value for a SM Higgs-boson of $500 \mathrm{GeV}$. 
fusion production cross section on the mixing angles for a heavy Higgs-boson mass of $500 \mathrm{GeV}$. The results are normalized to the corresponding SM Higgs-boson production cross section, and we observe that the cross section is maximum when $\sin (\beta-\alpha) \rightarrow 0$ and tends to vanish for $\sin (\beta-\alpha) \rightarrow \pm 1$. As the lightest Higgs boson $h^{0}$ has to be SM-like, $\sin (\beta-\alpha) \sim \pm 1$, some slight deviations being however still allowed by current measurements [57]. This constraint will nevertheless be omitted from our analysis in which we aim to determine the constraints on the parameter space that are issued solely from Higgs cascades at the LHC. The asymmetry of the cross section dependence on $\sin (\beta-\alpha)$ (relatively to $\sin (\beta-\alpha)=0$ ) originates from the $\alpha$ and $\beta$ dependence in Eq. (3.5). The cross section is hence enhanced both for small values of $\tan \beta$ (due to an enhancement of the contributions of the top-quark loops) and large values of $\tan \beta$ (due to an enhancement of the contributions of the bottom-quark loops). The top-loop enhancement is more pronounced in the positive $\sin (\beta-\alpha)$ half-plane, while the bottom-loop one impacts the negative $\sin (\beta-\alpha)$ half-plane. Moreover, any further increase of $\tan \beta$ beyond 20 does not lead to any appreciable effect via the bottom loops, so that we impose $\tan \beta<20$ in the following analysis.

The partial widths associated with the $H^{0} \rightarrow A Z$ and $A \rightarrow h^{0} Z$ decays are controlled by the scaling factors $\sin (\beta-\alpha)$ and $\cos (\beta-\alpha)$ respectively, as illustrated by Eq. (3.4). As a result, the intermediate region in which $\sin (\beta-\alpha)$ is different both from 0 and \pm 1 features an interesting enhancement of the $H^{0} \rightarrow h^{0} \mathrm{ZZ}$ decay. In the upper right and lower panels of Fig. 8, we present contours of specific branching ratios values for the $H^{0} \rightarrow A Z$ and $A \rightarrow h^{0} Z$ decays for representative Higgs-boson mass choices corresponding to the benchmark points introduced in Section II. As expected, we observe that the $H^{0} \rightarrow A Z$ decay becomes prominent for $\sin (\beta-\alpha) \sim \pm 1$, while the
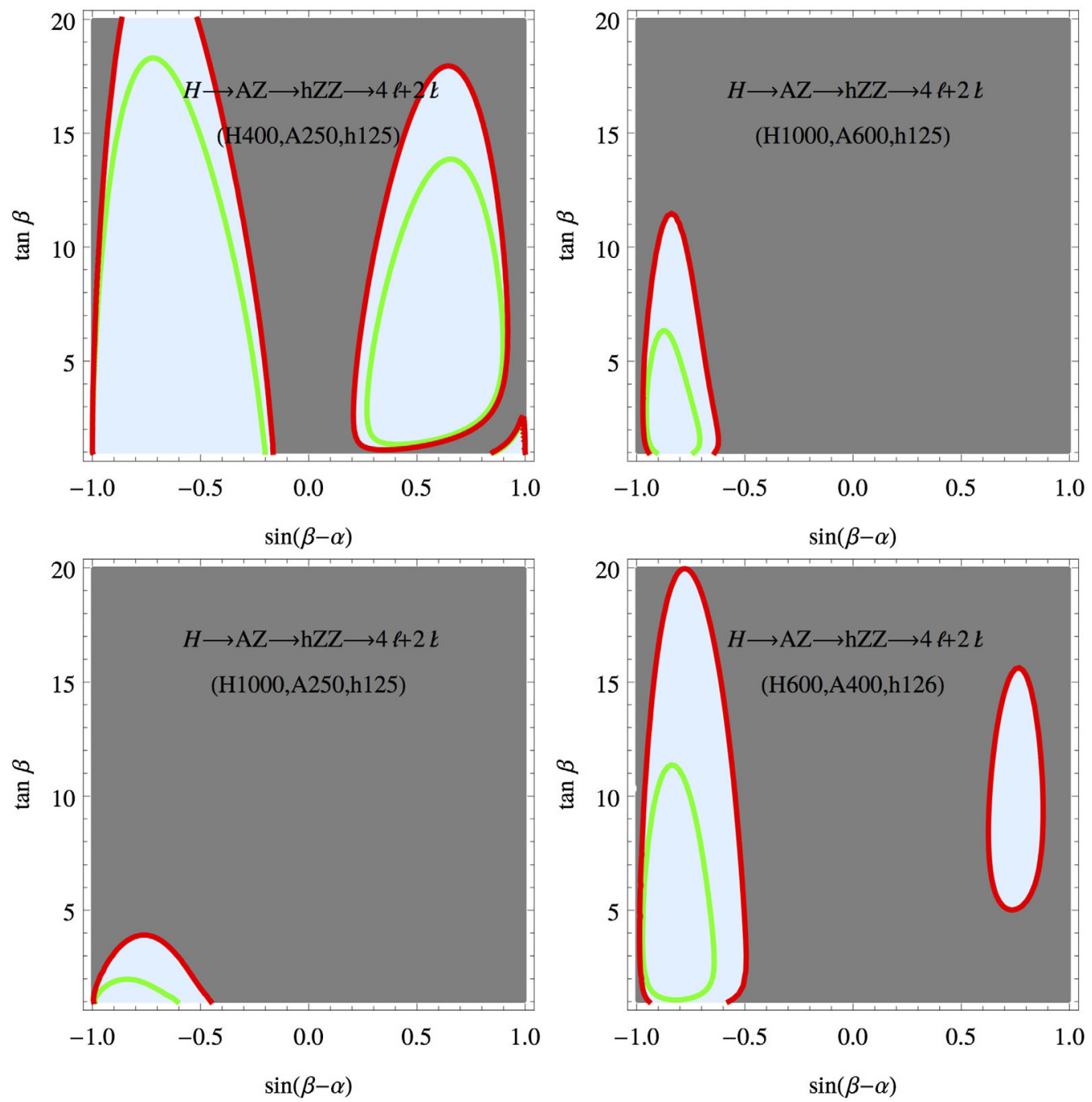

FIG. 9. Sensitivity of the LHC in the 2HMD parameter space once all Higgs masses have been fixed as in the BP1 (upper left), BP2 (upper right), BP3 (lower left) and BP4 (lower right) scenarios. The green and red contours respectively correspond to the region covered by the $N(b)=2$ and $N(b)=1$ signal regions for an integrated luminosity of $1000 \mathrm{fb}^{-1}$. 
$A \rightarrow h^{0} Z$ one exhibits a complementary behavior and becomes smaller in this region. The qualitative difference in the behavior of the pseudoscalar decay into a $h^{0} Z$ pair for the BP1-like (lower left panel) and BP2-like (lower right panel) configurations stems from the $t \bar{t}$ channel that is kinematically open in the BP2 case and is dominant for low values of $\tan \beta$. On the other hand, the decays into $b \bar{b}$ and $\tau^{+} \tau^{-}$systems are enhanced for larger $\tan \beta$ values, and the partial width of the $A \rightarrow h^{0} Z$ decay increases for $\sin (\beta-\alpha) \rightarrow 0$, as shown in Eq. (3.4). Consequently, the branching ratio associated with the $A \rightarrow h^{0} Z$ decay is bounded from above in the large $\tan \beta$ region for both scenarios, as well as for small $\tan \beta$ values in the BP2 case. This explains the origins of the closed contours of given branching ratio values obtained for the BP2 scenario. On different grounds, we have found that there is no qualitative differences across scenarios for the $H^{0} \rightarrow A Z$ branching ratio.

In Fig. 9, we show the allowed regions in the $(\sin (\beta-\alpha), \tan \beta)$ plane for mass configurations equal to those of the benchmark scenarios introduced in the former section. If the final-state topology is similar to the one encountered in the case of the considered benchmarks, the already-computed upper limits on the signal cross section could be applied. On the other hand, new limits could also be obtained after deriving the selection efficiency that would be associated with the new signal, following the different analysis strategies introduced in Sec. II.

The light blue regions shown on Fig. 9 correspond to parameter space configurations in which the cross section associated with the heavy Higgs-boson cascade process is large enough to yield a $5 \sigma$ discovery. The results are based on the numbers quoted in the previous section and are related to an integrated luminosity of $1000 \mathrm{fb}^{-1}$. The green contours determine the reach of the $N(b)=2$ signal region of the $4 \ell 2 b$ analysis while the red ones refer to the $N(b)=1$ signal region of the same analysis. As mentioned previously, the $4 b 2 \ell$ analysis is not considered as it is expected to lead to weaker bounds.

By virtue of a larger heavy-Higgs production cross section, the BP1 and BP4 scenarios are much better covered, the $H^{0}$ boson being indeed lighter than in the other cases. Moreover, while the functional form of the production cross section favors the $\sin (\beta-\alpha) \approx 0$ region, the product of the two branching ratio and their dependence on $\sin (\beta-\alpha)$ and $\cos (\beta-\alpha)$ moves the parameter space region of interest away from the $\sin (\beta-\alpha) \sim 0$ region. The bulk of the discovery reach is located, for the four benchmark, close to $\sin (\beta-\alpha) \sim 1$ that is precisely the region favored by current Higgs data. On the other hand, the dependence on $\tan \beta$ directly originates from the branching ratio results of Fig. 8 that show that large and small $\tan \beta$ values may respectively imply a reduced sensitivity due to the importance of the $A \rightarrow b \bar{b}$ decay and $A \rightarrow t \bar{t}$ decay (if relevant). While the rates of the cascade-decay processes undergone by heavier Higgs bosons may be suppressed, it is seen that they are indeed a viable option to find these additional Higgs bosons at the LHC particularly if they are moderately heavy. The expected suppression of the branching ratio has indeed been found not to be sufficient to balance the strength of simple selection cuts allowing for the separation of the signal from the background.

\section{CONCLUSIONS}

While the spectrum of the standard model has been established firmly today, physics beyond the Standard Model still remains a mystery. On the theoretical side, creative model building has explored avenues with an enlarged gauge group, extended matter representations and often a richer Higgs sector. While dedicated analyses are necessary to probe specific models of new physics, many models share common features (at least in terms of their spectra) so that they could be explored simultaneously in a general manner. In this spirit, this paper aims to study heavy neutral Higgs bosons that cascade decay into SM particles via intermediate lighter scalar states, as could occur in varied new physics theories. While exotic Higgsboson decays have been investigated in the literature, doubly-exotic modes involving several Higgs bosons have mostly not been targeted widely so far although they are an interesting probe for potential discovery.

In this work, we have discussed the generic cascade decay process $p p \rightarrow H_{2} \rightarrow H_{1} Z \rightarrow h^{0} \mathrm{ZZ}$ where a heavy Higgs boson $H_{2}$ decays into a lighter Higgs boson $H_{1}$ and a $Z$-boson, and where the $H_{1}$ boson further decays into a SM Higgs-boson $h^{0}$ and a $Z$-boson. Investigating a final-state signature made of either two $b$-jets and four charged leptons, or of four $b$-jets and two charged leptons, we have found that the discovery potential of such a process heavily depends on the magnitude of the mass splittings between the different scalar states, which directly impacts final-state object identification. It turned out that the $2 b 4 \ell$ channel is very promising, in particular when the requirement on the number of $b$-tagged jets is relaxed to $N(b)=1$. Although this channel does however not allow for the proper reconstruction of the heavy Higgs bosons, it provides an excellent handle for exhibiting the presence of a new physics signal. In contrast, the $4 b+2 \ell$ final state turns to be less promising, due to the non-perfect $b$-jet identification and the larger backgrounds.

We have begun with performing our collider analysis in a simplified-model approach inspired by the 2HDM, without resorting to specific values for the new physics couplings. This has allowed us to design several dedicated analyses, optimizing them for a good Higgs-cascade signal selection efficiency and an important associated background rejection. We have then applied our findings to assess the LHC discovery potential of a specific model that has been taken for the sake of the example to be the Type-II 2HDM. In this theoretical framework, we have found that the LHC is 
sensitive to Higgs-to-Higgs cascades in particular if the heaviest scalar state mass is moderate and for couplings close to those currently allowed by LHC Higgs data. This preferred configuration enhances on the one hand the heavy Higgs boson production cross section, and guarantees on the other hand that the decay products of the Higgs boson can properly be reconstructed. 2HDM compressed scenarios like our BP1 benchmark point satisfy both these criteria and are understandably expected to be better covered by future LHC results. Scenarios with a slightly heavier spectrum but exhibiting not too large mass splittings, like our BP4 scenario, are expected to be well probed too, however with a more limited reach. Finally, the sensitivity to scenarios like our BP2 and BP3 where the spectrum is much heavier (the heaviest state being at the $\mathrm{TeV}$ scale) is still appreciable but reduced as a consequence of the Higgs decay products being in a boosted regime for which our analysis is not sensitive to and the smaller production cross section.
Higgs cascades therefore offer a new channel to look for extended scalar sectors, complementing and potentially competing - at least in some models where heavier Higgs bosons for instance feature reduced couplings to fermions-with the more traditional approaches seeking heavier Higgs bosons.

\section{ACKNOWLEDGMENTS}

S. S. would like to thank J. Beuria and P. Sahoo for help with MADGRAPH and ROOT. B. C. acknowledges support from the Department of Science and Technology, India, under Grant No. YSS/2015/001771. The work of B. F. has been partly supported by French state funds managed by the Agence Nationale de la Recherche (ANR), in the context of the LABEX ILP (ANR-11-IDEX-0004-02, ANR-10-LABX-63). P.P. acknowledges the support of the SERB, DST of India Project No. (EMR/2015/000333).
[1] G. Aad et al. (ATLAS Collaboration), Observation of a new particle in the search for the standard model Higgs boson with the ATLAS detector at the LHC, Phys. Lett. B 716, 1 (2012).

[2] S. Chatrchyan et al. (CMS Collaboration), Observation of a new boson at a mass of $125 \mathrm{GeV}$ with the CMS experiment at the LHC, Phys. Lett. B 716, 30 (2012).

[3] G. Aad et al. (ATLAS, CMS Collaborations), Measurements of the Higgs boson production and decay rates and constraints on its couplings from a combined ATLAS and CMS analysis of the LHC pp collision data at $\sqrt{s}=7$ and $8 \mathrm{TeV}$, J. High Energy Phys. 08 (2016) 045.

[4] G. Aad et al. (ATLAS, CMS Collaborations), Combined Measurement of the Higgs Boson Mass in $p p$ Collisions at $\sqrt{s}=7$ and $8 \mathrm{TeV}$ with the ATLAS and CMS Experiments, Phys. Rev. Lett. 114, 191803 (2015).

[5] T. D. Lee, A theory of spontaneous T violation, Phys. Rev. D 8, 1226 (1973).

[6] G. C. Branco, P. M. Ferreira, L. Lavoura, M. N. Rebelo, M. Sher, and J. P. Silva, Theory and phenomenology of twoHiggs-doublet models, Phys. Rep. 516, 1 (2012).

[7] H. P. Nilles, Supersymmetry, supergravity and particle physics, Phys. Rep. 110, 1 (1984).

[8] H. E. Haber and G. L. Kane, The search for supersymmetry: Probing physics beyond the standard model, Phys. Rep. 117, 75 (1985).

[9] U. Ellwanger, C. Hugonie, and A. M. Teixeira, The next-tominimal supersymmetric standard model, Phys. Rep. 496, 1 (2010).

[10] S. Moretti, R. Santos, and P. Sharma, Optimising charged Higgs boson searches at the Large Hadron Collider across $b \bar{b} W^{ \pm}$final states, Phys. Lett. B 760, 697 (2016).
[11] A. Arhrib, R. Benbrik, and S. Moretti, Bosonic decays of charged Higgs bosons in a 2HDM Type-I, Eur. Phys. J. C 77, 621 (2017).

[12] B. Coleppa, F. Kling, and S. Su, Charged Higgs search via $A W^{ \pm} / H W^{ \pm}$channel, J. High Energy Phys. 12 (2014) 148.

[13] G. Aad et al. (ATLAS Collaboration), Search for a $C P$-odd Higgs boson decaying to $\mathrm{Zh}$ in pp collisions at $\sqrt{s}=8 \mathrm{TeV}$ with the ATLAS detector, Phys. Lett. B 744, 163 (2015).

[14] ATLAS Collaboration, Report No. ATLAS-CONF-2016015.

[15] ATLAS Collaboration, Report No. ATLAS-CONF-2017055.

[16] M. Aaboud et al. (ATLAS Collaboration), Search for heavy resonances decaying to a $W$ or $Z$ boson and a Higgs boson in the $q \bar{q}^{(\prime)} b \bar{b}$ final state in $p p$ collisions at $\sqrt{s}=13 \mathrm{TeV}$ with the ATLAS detector, Phys. Lett. B 774, 494 (2017).

[17] V. Khachatryan et al. (CMS Collaboration), Search for a pseudoscalar boson decaying into a $\mathrm{Z}$ boson and the $125 \mathrm{GeV}$ Higgs boson in $l^{+} l^{-} b \bar{b}$ final states, Phys. Lett. B 748, 221 (2015).

[18] V. Khachatryan et al. (CMS Collaboration), Search for neutral resonances decaying into a $\mathrm{Z}$ boson and a pair of $\mathrm{b}$ jets or $\tau$ leptons, Phys. Lett. B 759, 369 (2016).

[19] CMS Collaboration, Report No. CMS-PAS-HIG-16-010.

[20] S. Yang and Q.-S. Yan, Searching for heavy charged Higgs boson with jet substructure at the LHC, J. High Energy Phys. 02 (2012) 074.

[21] N. Chen, J. Li, Y. Liu, and Z. Liu, LHC searches for the $C P$-odd Higgs by the jet substructure analysis, Phys. Rev. D 91, 075002 (2015).

[22] B. Coleppa, F. Kling, and S. Su, Exotic decays of a heavy neutral Higgs through HZ/AZ channel, J. High Energy Phys. 09 (2014) 161. 
[23] B. Dumont, J. F. Gunion, Y. Jiang, and S. Kraml, Constraints on and future prospects for two-Higgs-doublet models in light of the LHC Higgs signal, Phys. Rev. D 90, 035021 (2014).

[24] J. Bernon, J. F. Gunion, H. E. Haber, Y. Jiang, and S. Kraml, Scrutinizing the alignment limit in two-Higgs-doublet models. II. $m_{H}=125 \mathrm{GeV}$, Phys. Rev. D 93, 035027 (2016).

[25] J. Bernon, J. F. Gunion, H. E. Haber, Y. Jiang, and S. Kraml, Scrutinizing the alignment limit in two-Higgs-doublet models: $m_{h}=125 \mathrm{GeV}$, Phys. Rev. D 92, 075004 (2015).

[26] F. Kling, A. Pyarelal, and S. Su, Light charged Higgs bosons to AW/HW via top decay, J. High Energy Phys. 11 (2015) 051.

[27] T. Li and S. Su, Exotic Higgs decay via charged Higgs, J. High Energy Phys. 11 (2015) 068.

[28] J. Li, R. Patrick, P. Sharma, and A. G. Williams, Boosting the charged Higgs search prospects using jet substructure at the LHC, J. High Energy Phys. 11 (2016) 164.

[29] R. Patrick, P. Sharma, and A. G. Williams, Exploring a heavy charged Higgs using jet substructure in a fully hadronic channel, Nucl. Phys. B917, 19 (2017).

[30] D. Goncalves and D. Lopez-Val, Pseudoscalar searches with dileptonic tops and jet substructure, Phys. Rev. D 94, 095005 (2016).

[31] F. Kling, J. M. No, and S. Su, Anatomy of exotic Higgs decays in 2HDM, J. High Energy Phys. 09 (2016) 093.

[32] N. Chakrabarty and B. Mukhopadhyaya, High-scale validity of a two Higgs doublet scenario: predicting collider signals, Phys. Rev. D 96, 035028 (2017).

[33] P. M. Ferreira, S. Liebler, and J. Wittbrodt, Wrong or right: $p p \rightarrow A \rightarrow Z h$ and the sign of the two-Higgs-doublet model, Phys. Rev. D 97, 055008 (2018).

[34] Z. Kang, J. Li, T. Li, D. Liu, and J. Shu, Probing the $C P$ even Higgs sector via $H_{3} \rightarrow H_{2} H_{1}$ in the natural next-tominimal supersymmetric standard model, Phys. Rev. D 88, 015006 (2013).

[35] J. Hajer, Y.-Y. Li, T. Liu, and J. F. H. Shiu, Heavy Higgs bosons at $14 \mathrm{TeV}$ and $100 \mathrm{TeV}$, J. High Energy Phys. 11 (2015) 124.

[36] M. Casolino, T. Farooque, A. Juste, T. Liu, and M. Spannowsky, Probing a light $C P$-odd scalar in di-topassociated production at the LHC, Eur. Phys. J. C 75, 498 (2015).

[37] E. Conte, B. Fuks, J. Guo, J. Li, and A. G. Williams, Investigating light NMSSM pseudoscalar states with boosted ditau tagging, J. High Energy Phys. 05 (2016) 100.

[38] R. Aggleton, D. Barducci, N.-E. Bomark, S. Moretti, and C. Shepherd-Themistocleous, Review of LHC experimental results on low mass bosons in multi Higgs models, J. High Energy Phys. 02 (2017) 035.

[39] U. Ellwanger and M. Rodriguez-Vazquez, Simultaneous search for extra light and heavy Higgs bosons via cascade decays, J. High Energy Phys. 11 (2017) 008.

[40] N. Craig, J. A. Evans, R. Gray, C. Kilic, M. Park, S. Somalwar, and S. Thomas, Multi-lepton signals of multiple Higgs bosons, J. High Energy Phys. 02 (2013) 033.
[41] C. Gao, M. A. Luty, M. Mulhearn, N. A. Neill, and Z. Wang, Searching for Additional Higgs Bosons via Higgs Cascades, arXiv:1604.03108.

[42] J. Alwall, R. Frederix, S. Frixione, V. Hirschi, F. Maltoni, O. Mattelaer, H.-S. Shao, T. Stelzer, P. Torrielli, and M. Zaro, The automated computation of tree-level and next-toleading order differential cross sections, and their matching to parton shower simulations, J. High Energy Phys. 07 (2014) 079.

[43] C. Degrande, C. Duhr, B. Fuks, D. Grellscheid, O. Mattelaer, and T. Reiter, UFO - The universal FeynRules output, Comput. Phys. Commun. 183, 1201 (2012).

[44] N. Christensen, P. de Aquino, C. Degrande, C. Duhr, B. Fuks, M. Herquet, F. Maltoni, and S. Schumann, A comprehensive approach to new physics simulations, Eur. Phys. J. C 71, 1541 (2011).

[45] A. Alloul, N. D. Christensen, C. Degrande, C. Duhr, and B. Fuks, FeynRules 2.0 - A complete toolbox for tree-level phenomenology, Comput. Phys. Commun. 185, 2250 (2014).

[46] R. D. Ball et al. (NNPDF Collaboration), Parton distributions for the LHC Run II, J. High Energy Phys. 04 (2015) 040 .

[47] T. Sjostrand, S. Mrenna, and P.Z. Skands, PYTHIA 6.4 physics and manual, J. High Energy Phys. 05 (2006) 026.

[48] J. de Favereau, C. Delaere, P. Demin, A. Giammanco, V. Lemaître, A. Mertens, and M. Selvaggi (DELPHES 3 Collaboration), DELPHES 3, A modular framework for fast simulation of a generic collider experiment, J. High Energy Phys. 02 (2014) 057.

[49] B. Dumont, B. Fuks, S. Kraml, S. Bein, G. Chalons, E. Conte, S. Kulkarni, D. Sengupta, and C. Wymant, Toward a public analysis database for LHC new physics searches using MADANALYSIS 5, Eur. Phys. J. C 75, 56 (2015).

[50] M. Cacciari, G. P. Salam, and G. Soyez, The anti-k(t) jet clustering algorithm, J. High Energy Phys. 04 (2008) 063.

[51] E. Conte, B. Fuks, and G. Serret, MadAnalysis 5, A userfriendly framework for collider phenomenology, Comput. Phys. Commun. 184, 222 (2013).

[52] E. Conte, B. Dumont, B. Fuks, and C. Wymant, Designing and recasting LHC analyses with MadAnalysis 5, Eur. Phys. J. C 74, 3103 (2014).

[53] M. Cacciari, G. P. Salam, and G. Soyez, FastJet user manual, Eur. Phys. J. C 72, 1896 (2012).

[54] G. Cowan, K. Cranmer, E. Gross, and O. Vitells, Asymptotic formulae for likelihood-based tests of new physics, Eur. Phys. J. C 71, 1554 (2011).

[55] S. Dittmaier et al. (LHC Higgs Cross Section Working Group Collaboration), Handbook of LHC Higgs cross sections: 1. Inclusive observables, arXiv:1101.0593.

[56] D. de Florian et al. (LHC Higgs Cross Section Working Group Collaboration), Handbook of LHC Higgs cross sections: 4. Deciphering the nature of the Higgs sector, arXiv:1610.07922.

[57] B. Coleppa, F. Kling, and S. Su, Constraining Type II 2HDM in light of LHC Higgs searches, J. High Energy Phys. 01 (2014) 161. 